\title{
CNTF protects neurons from hypoxic injury through the activation of STAT3 ${ }^{\text {pTyr705 }}$
}

\author{
YING LI GU ${ }^{1 *}$, GUAN QUN GAO ${ }^{*}$, NING MA ${ }^{2}$, LIN LIN YE $^{1}$, LI WEI ZHANG ${ }^{1}$, XU GAO $^{2}$ and ZHUO BO ZHANG ${ }^{1}$ \\ ${ }^{1}$ Department of Neurology, The Fourth Affiliated Hospital of Harbin Medical University, Harbin, Heilongjiang 150001; \\ ${ }^{2}$ Department of Biochemistry and Molecular Biology, Harbin Medical University, Harbin, Heilongjiang 150081, P.R. China
}

Received December 28, 2015; Accepted September 21, 2016

DOI: $10.3892 /$ ijmm.2016.2769

\begin{abstract}
The aim of the present study was to investigate whether ciliary neurotrophic factor (CNTF) plays its neuroprotective role following hypoxic injury through the activation of signal transducer and activator of transcription 3 (STAT3) signaling. Firstly, to determine whether CNTF exerts its effects via STAT3 following hypoxic injury, cultured neurons from the cerebral cortex of mice were prepared and a neuronal model of hypoxia was then established. The neurons exposed to hypoxia were then pre-treated with CNTF and transfected with small interference RNA (siRNA) targeting STAT3 (STAT3 siRNA) using polybrene, or with STAT3 ${ }^{\text {Tyr705 }}$ mutant or STAT3 ${ }^{\text {Ser727 }}$ mutant using an electroporation system. The survival, proliferation and neurite outgrowth of the neurons subjected to different treatments were also determined. RT-qPCR and western blot analysis were employed to examine the expression levels of STAT3, p-STAT3 ${ }^{\text {Tyr705 }}$ and p-STAT3 ${ }^{\text {Ser727 }}$ following treatment with CNTF and other treatments. Our results revealed that treatment with CNTF: i) protected neurons from hypoxic injury by promoting survival and neurite growth; ii) induced a significant increase in the levels of STAT3, STAT3 $3^{\text {pyr705 }}$ and the STAT3 ${ }^{\text {pTyr705}} /$ STAT3 ratio; it did not however, significantly affect the levels of STAT3 ${ }^{\text {Ser727 }}$ in the hypoxic cerebral cortex neurons. Transfection of the hypoxic neurons pre-treated with CNTF with STAT3 siRNA or STAT3 ${ }^{\text {Tyr705 }}$ neutralized the protective effects exerted by CNTF. The findings of our study thus demonstrate that $\mathrm{CNTF}$ protects neurons from hypoxic injury through the activation of STAT3 $3^{\text {pTyr705 }}$.
\end{abstract}

Correspondence to: Professor Xu Gao, Department of Biochemistry and Molecular Biology, Harbin Medical University, 194 Xuefu Road, Harbin, Heilongjiang 150081, P.R. China

E-mail: gaoxu20142015@126.com

Professor Zhuo Bo Zhang, Department of Neurology, The Fourth Affiliated Hospital of Harbin Medical University, 37 Yiyuan Street, Harbin, Heilongjiang 150001, P.R. China

E-mail: zzbzzx@163.com

*Contributed equally

Key words: hypoxia, ciliary neurotrophic factor, signal transducer and activator of transcription 3, neuron, STAT3 $3^{\text {pTyr705 }}$

\section{Introduction}

Neonatal hypoxia/ischemia and subsequent brain damage continue to be an alarming socio-sanitary problem, being considered the single-most important cause of acute mortality and chronic disability in newborns worldwide (1-3). Although improved obstetric and neonatal intensive care practices have led to increased survival, infants born very preterm are prone to disorders of cerebral development, including impaired cognition and behavior, epilepsy and cerebral palsy (4-6). Similarly, infants who suffer brain injury from hypoxia/ischemia during critical developmental periods of cerebral circuit formation are also at an increased risk of developing seizures, neuropsychiatric conditions and cognitive disorders (7). The severity of neonatal encephalopathy depends on the intensity, duration and location of the insult $(8,9)$. Approximately $15-20 \%$ of affected newborns will die in the post-natal period and an additional $25 \%$ will develop severe and permanent neuropsychological sequelae (10). Only are a small percentage of infants with severe injury survive without any handicaps $(10,11)$.

Ciliary neurotrophic factor (CNTF) is a member of NTF family originally isolated from chick embryo ciliary neurons, which: i) promote survival and/or differentiation in many cell types; and ii) have been demonstrated to have therapeutic potential in neurodegenerative diseases and the injured central nervous system (CNS) (12). CNTF exerts its biological functions by binding to high or low affinity receptor complexes consisting of CNTFR'gp130-LIFR or IL-6R'gp130·LIFR, respectively (13). Recent data indicate that distinct intracellular signaling pathways mediate diverse neuroprotective processes in response to CNTF. There is evidence to indicate that Janus kinase 2 (JAK2)/ signal transducer and activator of transcription 3 (STAT3), mitogen-activated protein kinase (MAPK)-extracellular signal-regulated protein kinase (ERK)1/2, as well as phosphatidylinositol-3-kinase (PI3K)/Akt, play important roles in promoting neuronal survival and process the outgrowth response to CNTF (14-17). STAT3 is known to modulate injury following an imbalance between pro- and anti-inflammatory cytokines in peripheral and CNS injury, rendering it a potential molecule for study. It has been demonstrated that CNTF plays a role in neural stem/progenitor cell (NSP) cell responses to hypoxia/ischemia (18). As a major transducer of CNTF-mediated neuroprotective activity, the activation of the JAK2/STAT3 axis by CNTF has been demonstrated to be 
responsible for the neuroprotective effects against the pathogenesis of Alzheimer's disease (AD) (14). It also points towards a significant role of STAT3 signaling following micro- and astrogliosis in the pathophysiology of neonatal hypoxia-related brain injury (19). However, whether the JAK/STAT3 axis mediated by CNTF is responsible for the neuroprotective effects in hypoxic injury remains unknown.

The aim of this study was to investigate whether CNTF plays its neuroprotective role following hypoxic injury through the activation of STAT3 signaling. Firstly, to determine whether CNTF exerts its effects via STAT3 following hypoxia, cultured neurons from the cerebral cortex of mice were prepared and a neuronal model of hypoxia was established. The neurons exposed to hypoxia were pre-treated with CNTF and transfected with small interference RNA targeting STAT3 (STAT3 siRNA) using polybrene, or with STAT3 ${ }^{\text {Tyr705 }}$ mutant or STAT3 ${ }^{\text {Ser727 }}$ mutant using an electroporation system. The survival, proliferation, and neurite outgrowth of neurons subjected to different treatments were also determined. Reverse transcription-quantitive PCR (RT-qPCR) and western blot analysis were employed to examine the expression levels of STAT3, p-STAT3 ${ }^{\text {Tyr705 }}$ and p-STAT3 ${ }^{\text {Ser727 following }}$ treatment with CNTF and other treatments.

\section{Materials and methods}

Ethics statement. Animal use and care were carried out in accordance with the animal care guidelines, which conformed to the Guide for the Care and Use of Laboratory Animals published by the US National Institutes of Health (NIH Publication no. 85-23, revised 1996). The Ethics Committee of Harbin Medical University specifically approved this study (Permit number: 2014ME1028). All efforts were made to minimize animal suffering.

Neuronal culture. Pregnant mice were housed with free access to food and water and exposed to a 12-h light/dark cycle at the Animal Central of Harbin Medical University, Harbin, China. A total of 20 neonatal C57BL/6J mice (during postnatal $24 \mathrm{~h}$ ), which were purchased from Harbin Medical University, were then employed in the present study for cell culture. The mice were sacrificed by decapitatation and disinfected in $75 \%$ ethanol. The hippocampus was then completely removed following craniotomy. The hippocampus was dissected into slices at a volume of $1 \mathrm{~mm}^{3}$ using an anatomic microscope in PBS and then digested for $30 \mathrm{~min}$ at $37^{\circ} \mathrm{C}$ incubator with $2 \mathrm{mg} / \mathrm{ml}$ papain (Roche Diagnostics $\mathrm{GmbH}$, Mannheim, Germany) containing $2 \mu \mathrm{l} / \mathrm{ml}$ DNAase. Termination of the digestion was performed by the addition of an equal amount of DMEM including $10 \%$ FBS and $1 \%$ penicillin-streptomycin solution. The cell suspension was centrifuged at 1,000 rpm for $10 \mathrm{~min}$ at $4^{\circ} \mathrm{C}$. After discarding the supernatant, the cells were re-suspended in the same medium by gently pipetting up and down and seeded on a 24-well plate at a density of $2 \times 10^{5}$ cells $/ \mathrm{ml}$. The medium was replaced with neurobasal medium (Gibco-BRL, Grand Island, NY, USA) supplemented with $2 \%$ B27 and 1\% penicillin-streptomycin solution.

Neuronal model of hypoxia. Hypoxia was achieved by placing the neurons in a modular incubator chamber (BillupsRothenberg, Del Mar, CA, USA) that was then flushed for 5 min
(20 $\mathrm{l} / \mathrm{min}$ ) with a gas mixture of $90 \% \mathrm{~N}_{2}, 5 \% \mathrm{CO}_{2}$ and $5 \% \mathrm{O}_{2}$. These conditions are reported by the manufacturer to render the hypoxia chamber completely purged.

Experimental grouping. The cultured neurons were divided into different groups as follows: i) the normal untreated neurons; ii) the hypoxia group (the neurons were exposed to hypoxia as described above); iii) the hypoxia + CNTF-treated group [CNTF (recombinant human CNTF; Novoprotein Scientific, Inc., Summit, NJ, USA) was added to the culture exposed to hypoxia at a concentration of $100 \mathrm{ng} / \mathrm{ml}$ (20)]; the iv) si-STAT3 group [neurons were exposed to hypoxia, treated with CNTF and transfected with STAT3 siRNA (hyp oxia + CNTF + STAT3 siRNA)]; v) the si-STAT3 control group [neurons were exposed to hypoxia, treated with $\mathrm{CNTF}$ and transfected with control siRNA (hypoxia + CNTF + control STAT3 siRNA; STAT3-si-Non)]; vi) the STAT3 ${ }^{\text {Tyr705 }}$ mutant group [neurons were exposed to hypoxia, treated with CNTF and transfected with STAT3 Y705F mutant (STAT3 ${ }^{\text {Tyr705 }}$ mutant) (hypoxia + CNTF + STAT3 ${ }^{\text {Tyr705 }}$ mutant)] ; vii) the STAT3 ${ }^{\text {Ser727 }}$ mutant group [neurons were exposed to hypoxia, treated with CNTF and transfected with STAT3 S727A mutant (STAT3 ${ }^{\text {Ser727 }}$

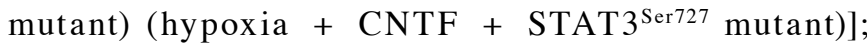
and viii) STAT3 mutant control group [neurons were exposed to hypoxia, treated with $\mathrm{CNTF}$ and transfecfed with the blank pcDNA3 vector (hypoxia + CNTF + blank vector pcDNA3)]. The STAT3 Y705F and S727A mutants, and pcDNA3 vector were purchased from Shenggong Biotechnology, Co. (Shanghai, China).

STAT3 gene knockdown. According to the CDS of STAT3 recorded in Nucleotide, we predesigned siRNA targeting the mouse STAT3 gene (GenBank accession no. U06922.1) using the online system RNAiDesigner (http://RNAiDesigner. invitrogen.com). The siRNA sequences targeting STAT3 were as follows: si-1, 5'-CCACGTTGGTGTTTCATAA-3'; si-2, 5'-GGGTGAAATTGACCAGCAA-3'; si-3, 5'-GCAGATG TTGGAGCAGCAT-3'; and si-4, 5'-CCAGATGCGGAGAA GATT-3'. A scrambled non-target siRNA was also used as a control (STAT3-si-Non). The lentivirus was packaged in PC12 cells (purchased from the Cell Bank of the Chinese Academy of Sciences, Shanghai, China) using Lipofectamine 2000 (Invitrogen Life Technologies, Carlsbad, CA, USA) and viral titers were determined. The interference efficiency of si-1-4 targeting STAT3 in the PC12 cells was determined by reverse transcription-quantitative PCR (RT-qPCR) and western blot analysis. The target siRNA-2 was selected for further investigation as it had the highest interference efficiency. The neuronal cells exposed to hypoxia were then infected with $1 \times 10^{6}$ recombinant lentivirus-transducing units containing the target siRNA or non-target siRNA in the presence of $6 \mu \mathrm{g} / \mathrm{ml}$ polybrene (Sigma-Aldrich, St. Louis, MO, USA), respectively.

$R T-q P C R$. The expression levels of STAT3 in the neuronal cells in each group were detected by RT-qPCR. RNA was extracted from the cells using TRIzol reagent (Invitrogen Life Technologies). Total RNA ( $2 \mu \mathrm{g})$ was used for cDNA synthesis using moloney murine leukemia virus reverse transcriptase (MMLV-RT; Takara Bio, Inc., Otsu, Japan), and the reverse transcript was used as the template for RT-qPCR using a Tower qRT-PCR system (Analytik Jena, Jena, Germany). qPCR was 
conducted using 2X Mix SYBR-Green I (Biosea Biotechnology, Co., Ltd., Beijing, China) (10 $\mu \mathrm{l})$, primer $(0.25 \mu \mathrm{l}$, $10 \mathrm{pmol} / \mathrm{l})$, template DNA $(1 \mu \mathrm{l})$ and sterile water $(8.5 \mu \mathrm{l})$. All PCR reactions included initial denaturation and multiple cycles at $\left(95^{\circ} \mathrm{C}\right.$ for $\left.3 \mathrm{~min}\right) ; 37$ cycles at $95^{\circ} \mathrm{C}$ for $10 \mathrm{sec}, 54^{\circ} \mathrm{C}$ for $10 \mathrm{sec}$, and $72^{\circ} \mathrm{C}$ for $30 \mathrm{sec}$; followed by $95^{\circ} \mathrm{C}$ for $10 \mathrm{sec}, 65^{\circ} \mathrm{C}$ for $5 \mathrm{sec}$ and a final $95^{\circ} \mathrm{C}$ for $15 \mathrm{sec}$. The primer for each gene was synthesized by Invitrogen Life Technologies. The qPCR primers used to quantify GAPDH expression were s follows: forward, 5'-CGAGATCCCTCCAAAATCAA-3' and reverse, 5'-TTCACACCCATGACGAACAT-3'; and for STAT3 forward, 5'-TCAGTGGAACCAGCTGCA-3' and reverse, 5'-AGAATC AAGCAGTTTCTG-3'. The expression of STAT3 was normalized to endogenous GAPDH expression. The $\mathrm{Ct}$ value was defined as the number of cycles required for the fluorescent signal to cross the threshold (i.e., exceed the background level). The correlation between the $\mathrm{Ct}$ value and the DNA copy number was calculated as follows: $\mathrm{Ct}=-3.347424 \mathrm{x} \log$ copy number +35.885406 , as previously described (21).

Transfection of neurons with STAT3 ${ }^{\text {Tyr705 }}$ mutant and STAT3 $3^{\text {Ser727 }}$ mutant. The Neon ${ }^{\mathrm{TM}}$ electroporation transfection system (Invitrogen, Eugene, OR, USA) was used to transfect the STAT3 mutant-pcDNA vector into the cultured neurons. Approximately 10 million cells were harvested, pelleted at $800 \mathrm{rpm}$ and washed with $1 \mathrm{X}$ Dulbecco's phosphate-buffered saline (DPBS containing $\mathrm{NaCl}, \mathrm{Na}_{2} \mathrm{PO}_{4}$ and $\mathrm{KCl}$, but not $\mathrm{Ca}^{2+}$ and $\mathrm{Mg}^{2+}$ ) prior to re-suspending in resuspension buffer $\mathrm{R}$, provided by the manufacturer. STAT3 mutants $(100 \mathrm{nM})$ were then mixed with the suspended neurons and loaded into a $100 \mu \mathrm{l}$ Neon tip. The neurons were then transfected with the STAT3 mutant-pcDNA vector via the Neon electroporation system at $1150 \mathrm{~V} / 30 \mathrm{msec}$ for 2 pulses. The cells were then cultured at $37^{\circ} \mathrm{C} / 5 \% \mathrm{CO}_{2} / 95 \%$ humidity for $48 \mathrm{~h}$ prior to harvesting and further analysis.

Western blot analysis. The STAT3 and p-STAT3 levels in the different groups were determined by western blot analysis. Briefly, the cells were lysed for $30 \mathrm{~min}$ in Cytobuster protein extraction buffer (Novagen, Madison, WI, USA) and centrifuged at $12,000 \mathrm{rpm}$. The supernatant was collected, total protein was measured, and $50 \mu \mathrm{g}$ were used for $10 \%$ sodium dodecyl sulfate-polyacrylamide gel electrophoresis (SDS-PAGE). The protein was then transferred to a nitrocellulose (NC) membrane and sealed with Tris-buffered saline and Tween-20 (TBST) containing 5\% non-fat milk powder. The membrane was subsequently incubated with goat anti-rat STAT3 $(1: 1,000)$ and rabbit anti-p-STAT3 (Tyr705, rabbit mAb no. 9145 and Ser727, mouse mAb no. 9136) antibodies (both from Cell Signaling Technology, Inc., Danvers, MA, USA), and mouse anti-rat GAPDH (1:500, sc-81545; Santa Cruz Biotechnology, Inc., Santa Cruz, CA, USA) antibody at $4^{\circ} \mathrm{C}$ overnight. After washing in TBST, the membrane was incubated with horseradish peroxidase (HRP)-conjugated secondary antibody (1:2,000; A0208; Beyotime Institute of Biotechnology, Shanghai, China) at $25^{\circ} \mathrm{C}$, and the protein quantity was determined using electrochemiluminescence (ECL) technique (Bestbio Biotechnology, Co., Ltd., Shanghai, China). The results were photographed using the JS gel imaging system and the grey density was calculated using SensiAnsys software (both from Shanghai Peiqing Science and Technology, Co., Ltd., Shanghai, China).
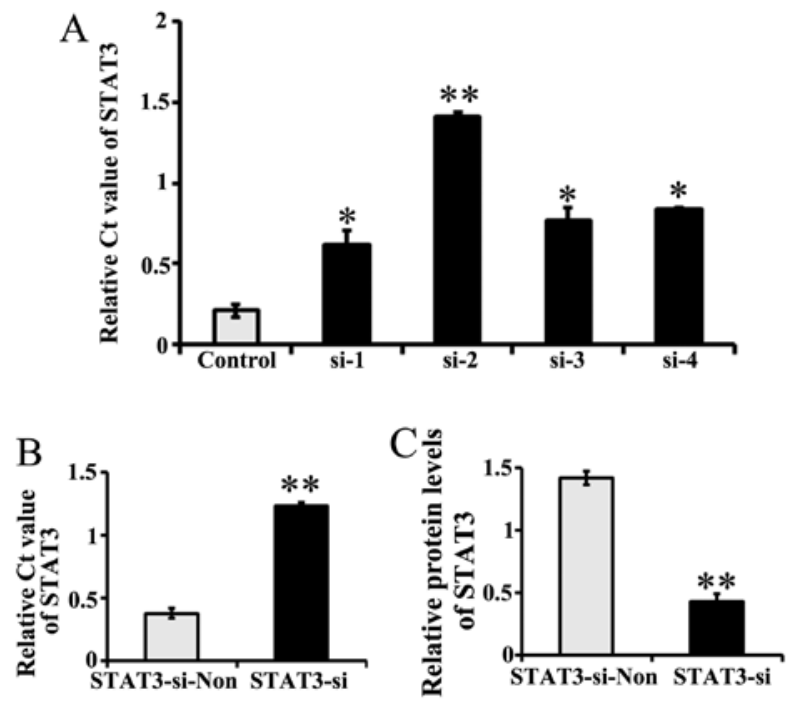

Figure 1. Expression of signal transducer and activator of transcription factor 3 (STAT3) in PC12 cells and cerebral cortex neurons following transfection with STAT3 siRNA. (A-C) Quantitative analysis of the relative mRNA and protein levels of STAT3 following transfection with STAT3 siRNA. GAPDH was used as an internal control. Considering the highest expression inhibition rates of STAT3, si-2 was selected as the target siRNA for use in further experiments. (A) PC12 cells; (B and C) cultured cerebral cortex neurons. Five independent experiments were performed. ${ }^{*} \mathrm{P}<0.05$, vs. control or STAT3-si-Non group; ${ }^{* *} \mathrm{P}<0.01$, vs. control or STAT3-si-Non group.

Determination of neuronal survival. Cell survival was evaluated by means of the trypan blue staining. In brief, cell numbers were determined by dispersing the neurons in trypsin and by counting using a coulter counter (model Z; Beckman Coulter, Palo Alto, CA, USA). These experiments were performed in triplicate in 24-well plates.

Assay for neurite outgrowth. For the evaluation of neurite outgrowth, thye cells treated as indicated and observed under a phase-contrast microscope (Leica DMi8; Leica Microsystems, Wetzlar, Germany) and the cell bodies and neurites were counted. The ratio between neurites and cell bodies was calculated yielding the average of neurites per neuron.

Statistical analysis. Data are presented as the means \pm SD. The comparisons of the mRNA levels and protein concentrations in the different groups were analyzed by one-way analysis of variance (ANOVA). Five independent experiments were performed. Statistical analyses were performed using GraphPad Prism, version 5.0 software (GraphPad Software, Inc., San Diego, CA, USA). A value of $\mathrm{P}<0.05$ was considered to indicate a statistically significant difference.

\section{Results}

Transfection with specific siRNA targeting STAT3 suppresses STAT3 expression. The stable transfection of 4 siRNAs targeting STAT3 (si-1, si-2, si-3 and si-4) in PC12 cells resulted in the inhibition of STAT3 expression by $>80 \%$ (Fig. 1A). Considering the highest expression inhibition rates observed for STAT3, si-2 was selected as the target siRNA for use in the following experiments. 

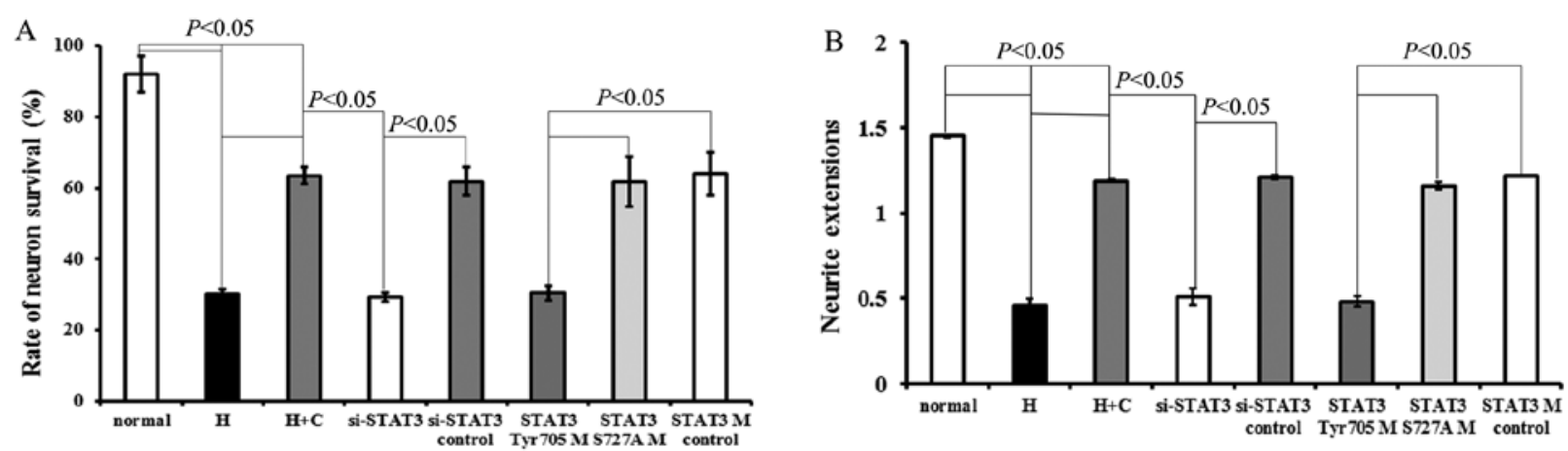

Figure 2. Effects of ciliary neurotrophic factor (CNTF) on survival and neurite growth of cultured neurons are mediated bhy signal transducer and activator of transcription factor 3 (STAT3) signaling. (A) Quantitative analysis of neuronal survival assessed by trypan blue staining. (B) Neurite outgrowth of neurons was evaluated in the different groups. The neurite length in neurons was evaluated following transfection with STAT3 siRNA, STAT3 ${ }^{\text {Tyr705 }}$ mutant or STAT3 ${ }^{\text {Ser727 }}$ mutant. Five independent experiments were performed. The values plotted are the means \pm SD.
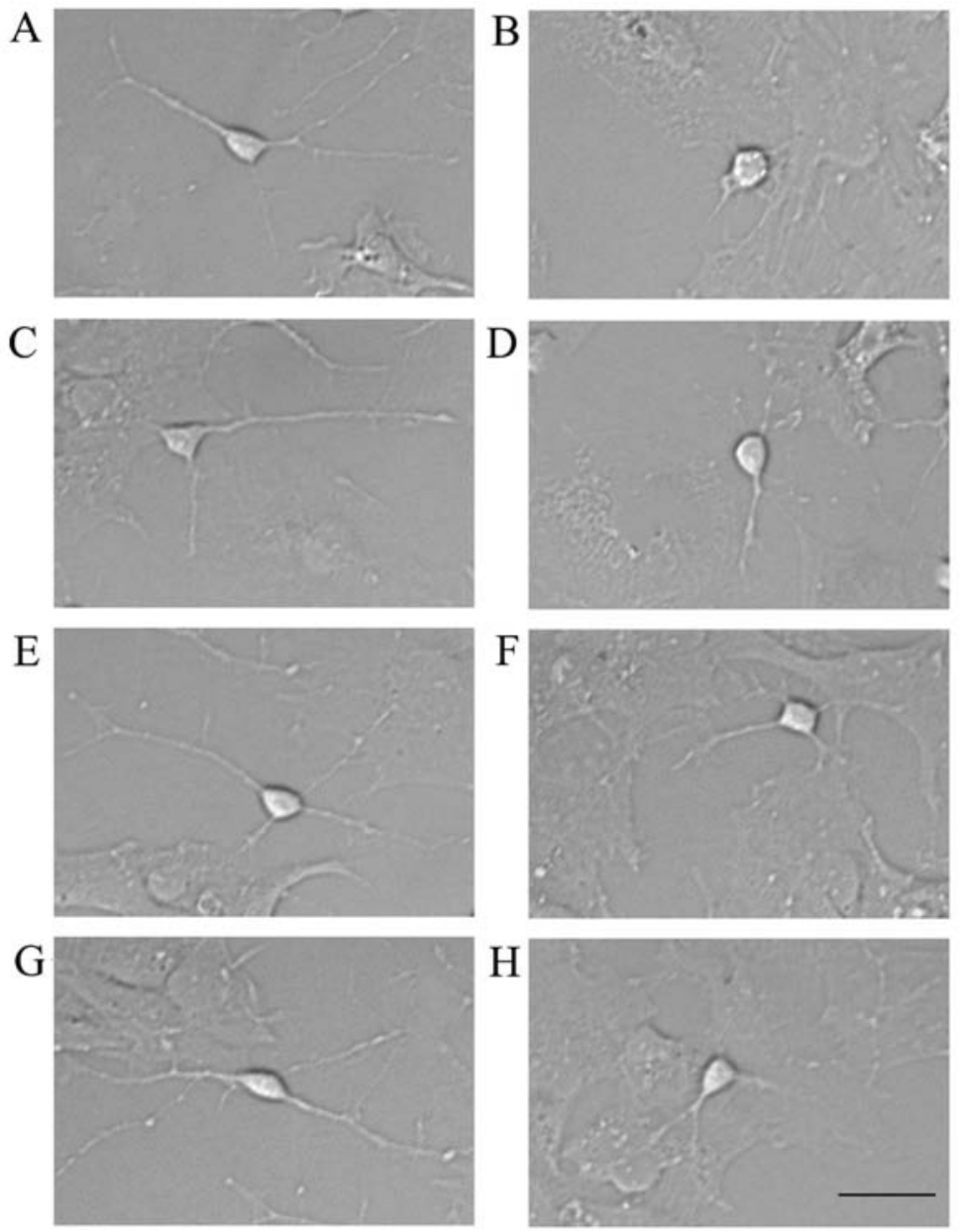

Figure 3. Morphology of cerebral cortex neurons transfected with different constructs. (A) Normal group; (B) hypoxia group; (C) hypoxia + CNTF; (D) STAT3 siRNA group; (E) si-STAT3 control group; (F) STAT3 ${ }^{\text {Tyr705 }}$ mutant group; (G) STAT3 ${ }^{\text {Ser727 }}$ mutant group; and (H) STAT3 mutant control group (magnification, x400). STAT3, signal transducer and activator of transcription factor 3. Five independent experiments were performed.

The cultured neurons were then stably transfected with STAT3 si-2 (named STAT3-si). Negative control neurons were transfected with non-target siRNA (recorded as STAT3si-Non). The STAT3 mRNA levels, as detected by RT-qPCR, were significantly lower (as indicated by the higher $\mathrm{Ct}$ value) in the STAT3 siRNA-transfected neurons than in the control siRNA-transfected ones ( $\mathrm{P}=0.00013, \mathrm{P}<0.01$; Fig. 1B). Western blotting found that the level of immunoreactive protein was significantly downregulated in STAT3-si-2 transfected neurons relative to the controls $(\mathrm{P}=0.00002, \mathrm{P}<0.01$; Fig. 1C).

Effects of CNTF on survival and neurite growth of neurons exposed to hypoxia. Exposure to hypoxia decreased the survival rate (vs. normal, $\mathrm{P}=0.00012, \mathrm{P}<0.05$; Fig. $2 \mathrm{~A}$ ) and neurite length of neurons (vs. normal, $\mathrm{P}=0.00012, \mathrm{P}<0.05$; Fig. 2B). The results revealed that CNTF had a significant protective effect on 


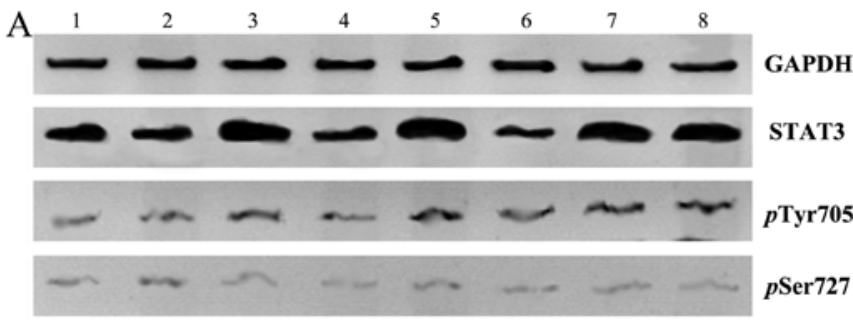

B

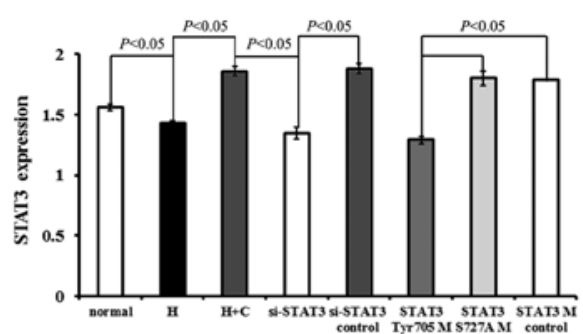

$\mathrm{C}$

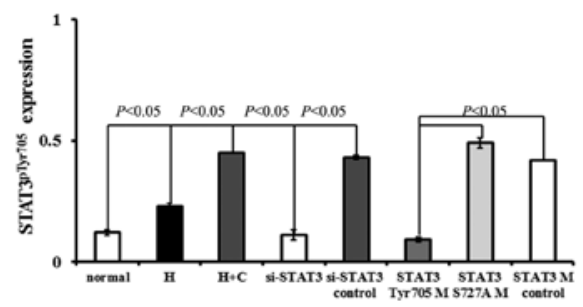

D

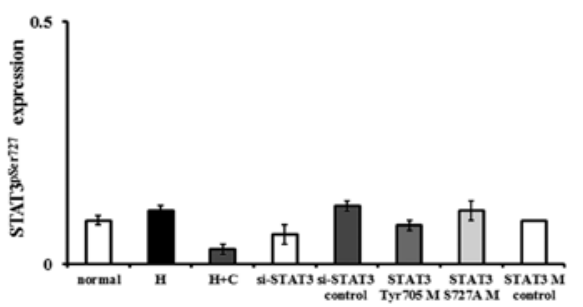

Figure 4. Ciliary neurotrophic factor (CNTF) exerts its effects via signal transducer and activator of transcription factor 3 (STAT3). (A) Expression of STAT3, STAT3 $3^{\text {pTyr705 }}$ and STAT3 ${ }^{\text {pSer727 }}$ in the different groups detected by western blot analysis. Lane 1, normal group; lane 2, hypoxia group; lane 3 , hypoxia + CNTF group; lane 4, si-STAT3 group; lane 5, si-STAT3 control group; lane 6 , STAT3 ${ }^{\mathrm{Ty} 705}$ mutant group; lane $7, \mathrm{STAT}^{3 \mathrm{Se} 727}$ mutant group; and lane 8, STAT3 mutant control group. (B-D) Quantitative analysis of the relative protein levels of STAT3, STAT3 $3^{\text {pTyr705 }}$ and STAT3 $3^{\text {per727 }}$ in the different groups determined by western blot analysis. GAPDH was used as an internal control. Five independent experiments were performed. The values plotted are the means $\pm \mathrm{SD}$.

neuronal survival under hypoxic conditions (Fig. 2A). In addition, the effects of CNTF on neurite growth were investigated. Following culture with CNTF for $48 \mathrm{~h}$, the hypoxia-exposed neurons (hypoxia + CNTF) displayed outgrowth in the form of neurite extensions, when compared with that of the hypoxiaexposed neurons not treated with $\mathrm{CNTF}(\mathrm{P}=0.0003, \mathrm{P}<0.05$; Figs. 2B and 3).

Transfection of the hypoxia-exposed neurons with STAT3 siRNA or STAT3 ${ }^{\text {Tyr705 }}$ mutant neutralized the protective effects induced by treatment with CNTF (compared with the si-STAT3 control group, $\mathrm{P}=0.0015, \mathrm{P}<0.05$; or compared with the STAT3 mutant control group, $\mathrm{P}=0.00025$, $\mathrm{P}<0.05$ ) (Figs. 2 and 3).

There was no significant difference between the STAT3 mutant control group and the si-STAT3 control group $(\mathrm{P}>0.05)$. Neither the STAT3 mutant control group nor the si-STAT3 control group exhibited a significant difference compared

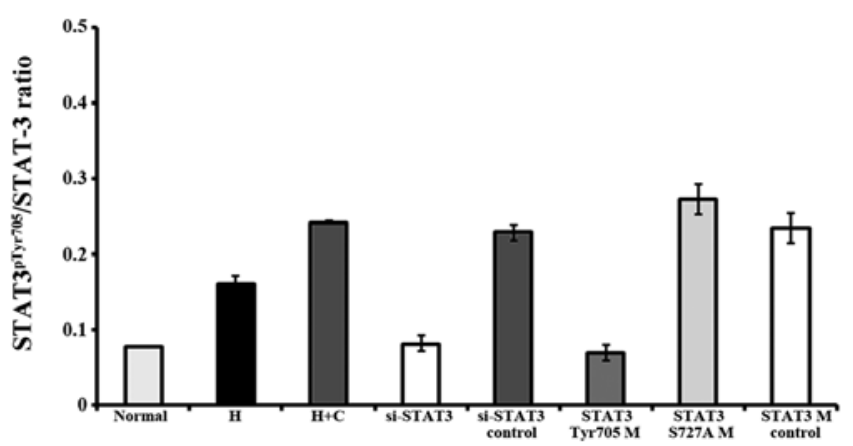

Figure 5. Signal transducer and activator of transcription factor 3 (STAT3 $3^{\text {pTyr705}) / ~}$ STAT3 ratio in the different groups. The ratio of STAT3 ${ }^{\text {Tyr705/STAT3 in the }}$ different groups detected by western blot analysis. Five independent experiments were performed. The values plotted are the means \pm SD.

with the hypoxia $+\mathrm{CNTF}$ group $(\mathrm{P}>0.05)$. Transfection with STAT3 ${ }^{\text {Ser727 }}$ mutant did not exert any exert any significant effecft on survival or neurite outgrowth compared to the hypoxia-exposed neurons or to the neurons transfected with the control siRNA or mutant (Fig. 2 and 3). Thus, our results suggest that CNTF protects neurons from hypoxic injury through STAT3 ${ }^{\mathrm{Tyr} 705}$, but not through STAT3 ${ }^{\text {Ser727. }}$

CNTF exerts protective effects against hypoxic injury to neurons through STAT3. As previously demonstrated, STAT3 ${ }^{\text {Tyr705 }}$ is an indicator of its transcriptional activation (22-24). In this study, to gain further insight into the association between STAT3 activation and CNTF treatment, recombinant human CNTF was used to treat neurons and the activation state of

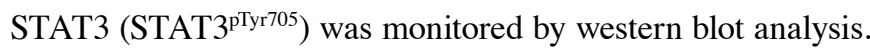
The level of STAT3 $3^{\text {pSer727 }}$ was also detected.

Treatment with CNTF induced a significant increase in the levels of STAT3 and STAT3 $3^{\text {Tyr705 }}$, and in the STAT3 $3^{\text {Tyr705/ }}$ STAT3 ratio, but not in the levels of STAT $3^{\text {per727 in the cerebral }}$ cortex neurons under hypoxic conditions (hypoxia $+\mathrm{CNTF}$ group vs. hypoxia group, $\mathrm{P}=0.00017, \mathrm{P}<0.05$ ) (Figs. 4 and 5).

Moreover, the blocking of STAT3 signaling by STAT3 siRNA in the neurons exposed to hypoxia and treated with CNTF (si-STAT3 group) prevented the CNTF-induced increase in the levels of STAT3 ${ }^{\text {pTyr705 }}$ (Fig. 4). Conversely, transfection with STAT3 ${ }^{\text {Tyr705 }}$ mutant suppressed STAT3 signaling which was activated in the neurons treated with CNTF (STAT3 ${ }^{\text {Tyr705 }}$ mutant group vs. STAT3 mutant control group, $\mathrm{P}=0.0006, \mathrm{P}<0.05)$. However, the above-mentioned suppressive effects were not observed in the neurons trans-

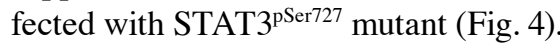

There was no significant difference between the STAT3 mutant control group and the si-STAT3 control group ( $\mathrm{P}>0.05)$. Neither the STAT3 mutant control group nor the si-STAT3 control group exhibited a significant difference compared with the hypoxia $+\mathrm{CNTF}$ group $(\mathrm{P}>0.05)$.

\section{Discussion}

The present data revealed that treatment with CNTF: i) protected neurons from hypoxic injury by promoting survival and neurite growth; ii) induced a significant increase in the 
levels of STAT3 and STAT3 ${ }^{\text {Pyr705 }}$, and in the STAT3 ${ }^{\text {pTyr705/ }}$ STAT3 ratio, but not in the levels of STAT $3^{\text {pSer727 in hypoxic }}$ cerebral cortex neurons. The blocking of STAT3 signaling using STAT3 siRNA prevented the CNTF-induced increase in the levels of STAT3 ${ }^{\text {pTyr705 }}$. Transfection of the hypoxic neurons treated with CNTF with STAT3 siRNA or STAT3 ${ }^{\text {Tyr705 }}$ mutant neutralized the protective effects exerted by CNTF. These results demonstrated that $\mathrm{CNTF}$ exerted neuroprotective effects against hypoxic injury through the activation of STAT3 $3^{\text {pyr705 }}$.

Our data demonstrated that treatment with CNTF protected the neurons from hypoxic injury by promoting survival rate and neurite growth. Hypoxia-associated brain damage results in immediate neuronal injury and in the exhaustion of cellular energy stores, which lead to a multi-faceted cascade of biochemical events, biological injury and neuronal death $(25,26)$. NTFs are essential proteins for the maintenance and survival of neurons in both developing and mature nervous systems $(27,28)$. Currently, CNTF is the only known factor which shows direct trophic effects on muscle and nerve system, and may have therapeutic effects on motor neuron diseases, nerve damage and muscular atrophy (29). Our data confirmed the fact that CNTF is an important neurocytokine for the survival and neurite growth of neurons following hypoxic injury.

Further experiments revealed that CNTF induced the phosphorylation of STAT3 in neurons under hypoxic conditions; however, the promoting effects of CNTF on survival and neurite growth of neuron was attenuated by transfection with STAT3 siRNA or STAT3 ${ }^{\text {Tyr705 }}$ mutant, but not by transfection with STAT3 ${ }^{\text {Ser727 }}$ mutant. These data demonstrated that CNTF exerted neuroprotective effects under hypoxic conditions through the activation of STAT3/STAT3 ${ }^{\text {Tyr705. It has been }}$ demonstrated that the cellular response to $\mathrm{CNTF}$ is mediated by a receptor complex consisting of the signal transducers glycoprotein 130 (gp130) and LIF receptor $\beta$ ( $\beta$-receptor components) and CNTFR $\alpha$ (30-32). The dimerization of the $\beta$-receptor components results in the phosphorylation of JAK (33) followed by signal transduction, including the STAT proteins $(32,33)$. The JAK/STAT pathway is considered to be the primary cytokine signaling pathway among other pathways, such as the Ras-mitogen-activated protein (Ras-MAP) kinase pathway, including ERK1 and ERK2 (MAPK/ERK kinase system) and the cell line-dependent PI3K pathway (PI3K/Akt system) $(32,34)$. It has been established that the JAK2/STAT3 pathway is mainly involved inthe survival of neurons in response to CNTF $(35,36)$. It has also been demonstrated that the STAT3 and PI3K/Akt pathways, but not the MEK/MAPK signaling play a major role in mediating the survival response of neurons by cytokines (37) with STAT3, specifically activated by CNTF, leading to increased neuronal survival (38). Phosphorylated STAT3 dimerizes and translocates to the nucleus to regulate target gene transcription (39). In addition, CNTF can also trigger and activate the PI3K/Akt or MEK/ERK pathways, either concomitantly or independently of the JAK2/STAT3 signaling pathway $(40,41)$. Moreover, STAT3 is phosphorylated at Tyr705 upon the activation of cytokine and growth factor receptors, resulting in its homodimerization and nuclear translocation to activate the transcription of downstream responsive genes $(22,24)$. Once activated, STAT3 mediates multiple biological functions, including the promotion of cell proliferation, angiogenesis and metastasis, the inhibition of differentiation and antitumor immune responses (42-44). Given these, the present study indicated that the protective roles of CNTF were dependent on STAT3/STAT3 $3^{\text {Pyr705}}$-mediated neuronal survival and proliferation under hypoxic conditions.

In conclusion, the findings of our study demonstrated that the treatment of neurons exposed to hypoxia with CNTF: i) protected cultured neurons from hypoxic injury by promotion survival and neurite growth; ii) induced the phosphorylation of STAT3. However, the promoting effects of CNTF on survival and neurite growth of neurons were suppressed by transfection with STAT3 siRNA or STAT3 ${ }^{\text {Tyr705 }}$ mutant, but not by transfection with STAT3 ${ }^{\text {Ser727 }}$ mutant. Taken together, the findings of the present study demonstrate that CNTF-mediated neuron survival and proliferation under hypoxic conditions is mediated by the activation of STAT3/ STAT3 $3^{\text {Tyr705. }}$.

\section{Acknowledgements}

This study was supported by the Health and Family Planning Commission of Heilongjiang Province (no. 2013097). We would like to thank the Labreal Bioscience and Technology, Ltd., Co., Kunming, China for their valuable contribution to parts of the experimental design.

\section{References}

1. du Plessis AJ and Volpe JJ: Perinatal brain injury in the preterm and term newborn. Curr Opin Neurol 15: 151-157, 2002.

2. Azra Haider B and Bhutta ZA: Birth asphyxia in developing countries: current status and public health implications. Curr Probl Pediatr Adolesc Health Care 36: 178-188, 2006.

3. Jiang H, Lei JJ and Zhang YH: Protective effect of topiramate on hypoxic-ischemic brain injury in neonatal rat. Asian Pac J Trop Med 7: 496-500, 2014

4. Marín-Padilla M: Perinatal brain damage, cortical reorganization (acquired cortical dysplasias), and epilepsy. Adv Neurol 84: 153-172, 2000.

5. Robinson S: Systemic prenatal insults disrupt telencephalon development: implications for potential interventions. Epilepsy Behav 7: 345-363, 2005.

6. Volpe JJ: Brain injury in premature infants: a complex amalgam of destructive and developmental disturbances. Lancet Neurol 8: 110-124, 2009.

7. Martinez-Biarge M, Diez-Sebastian J, Rutherford MA and Cowan FM: Outcomes after central grey matter injury in term perinatal hypoxic-ischaemic encephalopathy. Early Hum Dev 86: 675-682, 2010.

8. Ferriero DM: Neonatal brain injury. N Engl J Med 351: 1985-1995, 2004.

9. Juul SE and Ferriero DM: Pharmacologic neuroprotective strategies in neonatal brain injury. Clin Perinatol 41: 119-131, 2014.

10. Levene ML, Kornberg $\mathbf{J}$ and Williams TH: The incidence and severity of post-asphyxial encephalopathy in full-term infants. Early Hum Dev 11: 21-26, 1985.

11. Cerio FG, Lara-Celador I, Álvarez A and Hilario E: Neuroprotective therapies after perinatal hypoxic-ischemic brain injury. Brain Sci 3: 191-214, 2013.

12. Skaper SD, Selak I, Manthorpe M and Varon S: Chemically defined requirements for the survival of cultured 8-day chick embryo ciliary ganglion neurons. Brain Res 302: 281-290, 1984.

13. Wagener EM, Aurich M, Aparicio-Siegmund S, Floss DM, Garbers C, Breusing K, Rabe B, Schwanbeck R, Grötzinger J, Rose-John S and Scheller J: The amino acid exchange R28E in ciliary neurotrophic factor (CNTF) abrogates interleukin-6 receptor-dependent but retains $\mathrm{CNTF}$ receptor-dependent signaling via glycoprotein 130 (gp130)/leukemia inhibitory factor receptor (LIFR). J Biol Chem 289: 18442-18450, 2014. 
14. Wang K, Zhou F, Zhu X, Zhang K, Huang B, Zhu L and Zhu L Neuroprotective properties of ciliary neurotrophic factor on retinoic acid (RA)-predifferentiated SH-SY5Y neuroblastoma cells. Folia Neuropathol 52: 121-127, 2014.

15. Askvig JM and Watt JA: The MAPK and PI3K pathways mediate CNTF-induced neuronal survival and process outgrowth in hypothalamic organotypic cultures. J Cell Commun Signal 9: 217-231, 2015.

16. Zhou Q, Chen P, Di G, Zhang Y, Wang Y, Qi X, Duan H and Xie L: Ciliary neurotrophic factor promotes the activation of corneal epithelial stem/progenitor cells and accelerates corneal epithelial wound healing. Stem Cells 33: 1566-1576, 2015.

17. Severi I, Senzacqua M, Mondini E, Fazioli F, Cinti S and Giordano A: Activation of transcription factors STAT1 and STAT5 in the mouse median eminence after systemic ciliary neurotrophic factor administration. Brain Res 1622: 217-229, 2015.

18. Covey MV and Levison SW: Leukemia inhibitory factor participates in the expansion of neural stem/progenitors after perinatal hypoxia/ischemia. Neuroscience 148: 501-509, 2007.

19. Shrivastava K, Llovera G, Recasens M, Chertoff M, GiménezLlort L, Gonzalez B and Acarin L: Temporal expression of cytokines and signal transducer and activator of transcription factor 3 activation after neonatal hypoxia/ischemia in mice. Dev Neurosci 35: 212-225, 2013.

20. Schwieger J, Warnecke A, Lenarz T, Esser KH and Scheper V: Neuronal survival, morphology and outgrowth of spiral ganglion neurons using a defined growth factor combination. PLoS One 10: e0133680, 2015.

21. Lin JC, Xing YL, Xu WM, Li M, Bo P, Niu YY and Zhang CR: Evaluation of galactomannan enzyme immunoassay and quantitative real-time PCR for the diagnosis of invasive pulmonary aspergillosis in a rat model. J Microbiol Biotechnol 24: 1044-1050, 2014.

22. Quesnelle KM, Boehm AL and Grandis JR: STAT-mediated EGFR signaling in cancer. J Cell Biochem 102: 311-319, 2007.

23. Frank DA: STAT3 as a central mediator of neoplastic cellular transformation. Cancer Lett 251: 199-210, 2007.

24. Germain D and Frank DA: Targeting the cytoplasmic and nuclear functions of signal transducers and activators of transcription 3 for cancer therapy. Clin Cancer Res 13: 5665-5669, 2007.

25. Northington FJ, Chavez-Valdez R and Martin LJ: Neuronal cell death in neonatal hypoxia-ischemia. Ann Neurol 69: 743-758, 2011.

26. Li X, Zhang J, Chai S and Wang X: Progesterone alleviates hypoxic-ischemic brain injury via the Akt/GSK-3 $\beta$ signaling pathway. Exp Ther Med 8: 1241-1246, 2014.

27. Linker R, Gold R and Luhder F: Function of neurotrophic factors beyond the nervous system: inflammation and autoimmune demyelination. Crit Rev Immunol 29: 43-68, 2009.

28. Maisonpierre PC, Belluscio L, Squinto S, Ip NY, Furth ME, Lindsay RM and Yancopoulos GD: Neurotrophin-3: a neurotrophic factor related to NGF and BDNF. Science 247: 1446-1451, 1990.

29. Davis S, Aldrich TH, Valenzuela DM, Wong VV, Furth ME, Squinto SP and Yancopoulos GD: The receptor for ciliary neurotrophic factor. Science 253: 59-63, 1991.
30. Ip NY, Nye SH, Boulton TG, Davis S, Taga T, Li Y, Birren SJ, Yasukawa K, Kishimoto T, Anderson DJ, et al: CNTF and LIF act on neuronal cells via shared signaling pathways that involve the IL-6 signal transducing receptor component gp130. Cell 69: $1121-1132,1992$.

31. Sleeman MW, Anderson KD, Lambert PD, Yancopoulos GD and Wiegand SJ: The ciliary neurotrophic factor and its receptor, CNTFR alpha. Pharm Acta Helv 74: 265-272, 2000.

32. Turnley AM and Bartlett PF: Cytokines that signal through the leukemia inhibitory factor receptor-beta complex in the nervous system. J Neurochem 74: 889-899, 2000.

33. Stahl N, Boulton TG, Farruggella T, Ip NY, Davis S, Witthuhn BA, Quelle FW, Silvennoinen O, Barbieri G, Pellegrini S, et al: Association and activation of Jak-Tyk kinases by CNTF-LIF-OSM-IL-6 beta receptor components. Science 263: 92-95, 1994.

34. Boulton TG, Stahl N and Yancopoulos GD: Ciliary neurotrophic factor/leukemia inhibitory factor/interleukin 6/oncostatin M family of cytokines induces tyrosine phosphorylation of a common set of proteins overlapping those induced by other cytokines and growth factors. J Biol Chem 269: 11648-11655, 1994.

35. Kaur N, Kim IJ, Higgins D and Halvorsen SW: Induction of an interferon- $\gamma$ Stat 3 response in nerve cells by pre-treatment with gp130 cytokines. J Neurochem 87: 437-447, 2003.

36. Kaur N, Wohlhueter AL and Halvorsen SW: Activation and inactivation of signal transducers and activators of transcription by ciliary neurotrophic factor in neuroblastoma cells. Cell Signal 14: 419-429, 2002.

37. Alonzi T, Middleton G, Wyatt S, Buchman V, Betz UA, Müller W, Musiani P, Poli V and Davies AM: Role of STAT3 and PI 3-kinase/Akt in mediating the survival actions of cytokines on sensory neurons. Mol Cell Neurosci 18: 270-282, 2001.

38. Schweizer U, Gunnersen J, Karch C, Wiese S, Holtmann B, Takeda K, Akira S and Sendtner M: Conditional gene ablation of Stat 3 reveals differential signaling requirements for survival of motoneurons during development and after nerve injury in the adult. J Cell Biol 156: 287-297, 2002.

39. Zhong Z, Wen Z and Darnell JE Jr: Stat3: a STAT family member activated by tyrosine phosphorylation in response to epidermal growth factor and interleukin-6. Science 264: 95-98, 1994.

40. Sango K, Yanagisawa H, Komuta Y, Si Y and Kawano H: Neuroprotective properties of ciliary neurotrophic factor for cultured adult rat dorsal root ganglion neurons. Histochem Cell Biol 130: 669-679, 2008.

41. Rhee KD, Goureau O, Chen S and Yang XJ: Cytokine-induced activation of signal transducer and activator of transcription in photoreceptor precursors regulates rod differentiation in the developing mouse retina. J Neurosci 24: 9779-9788, 2004.

42. Leeman RJ, Lui VW and Grandis JR: STAT3 as a therapeutic target in head and neck cancer. Expert Opin Biol Ther 6: 231-241, 2006.

43. Johnston PA and Grandis JR: STAT3 signaling: anticancer strategies and challenges. Mol Interv 11: 18-26, 2011.

44. Regis G, Pensa S, Boselli D, Novelli F and Poli V: Ups and downs: the STAT1:STAT3 seesaw of interferon and gp130 receptor signalling. Semin Cell Dev Biol 19: 351-359, 2008. 